\title{
On the calculation of time correlation functions by potential scaling
}

\author{
Chenyue Xing and Ioan Andricioaei ${ }^{\mathrm{a})}$ \\ Department of Chemistry and The Program in Bioinformatics, University of Michigan, Ann Arbor, Michigan \\ 48109
}

(Received 4 October 2005; accepted 29 November 2005; published online 19 January 2006)

\begin{abstract}
We present and analyze a general method to calculate time correlation functions from molecular dynamics on scaled potentials for complex systems for which simulation is affected by broken ergodicity. Depending on the value of the scaling factor, correlations can be calculated for times that can be orders of magnitude longer than those accessible to direct simulations. We show that the exact value of the time correlation functions of the original system (i.e., with unscaled potential) can be obtained, in principle, using an action-reweighting scheme based on a stochastic path-integral formalism. Two tests (involving a bistable potential model and a dipeptide bond-vector orientational relaxation) are exemplified to showcase the strengths, as well as the limitations of the approach, and a procedure for the estimation of the time-dependent standard deviation error is outlined. (C) 2006 American Institute of Physics. [DOI: 10.1063/1.2159476]
\end{abstract}

\section{INTRODUCTION}

In the realm of kinetics, time correlation functions play the same central role as partition functions do for thermodynamics. Given the knowledge of the partition function, all thermodynamic functions (such as the free energy, entropy, heat capacity) can be calculated. Similarly, from the time correlation function of select microscopic variables one can compute, through the Green-Kubo formalism, bulk relaxation properties. Correlation functions therefore are instrumental to the theoretical description of a broad range of time-dependent topics such as transport coefficients, ${ }^{1}$ chemical reaction kinetics, ${ }^{2}$ dynamical light scattering, ${ }^{3}$ infrared absorption, ${ }^{4}$ Raman scattering, ${ }^{5}$ dielectric relaxation, ${ }^{6}$ NMR line shapes, ${ }^{7} \quad$ fluorescence depolarization, ${ }^{8}$ sound attenuation, ${ }^{9}$ etc.

Unfortunately, time correlation functions and partition functions not only share the limelight that makes them central to kinetics and thermodynamics respectively, but they also share a notorious difficulty in being computed (when the system is large). ${ }^{10}$ The classical equilibrium time correlation function of a property $A(\boldsymbol{\Gamma}(t))$ depending on the phase-space vector $\boldsymbol{\Gamma}=(x, p)$ of positions $x$ and momenta $p$ is, by definition,

$$
C(t)=\left\langle A(\boldsymbol{\Gamma}(0) A(\boldsymbol{\Gamma}(t))\rangle=\int A(\boldsymbol{\Gamma}(0)) A(\boldsymbol{\Gamma}(t)) f(\boldsymbol{\Gamma}) d \boldsymbol{\Gamma},\right.
$$

where $f(\boldsymbol{\Gamma})$ is the equilibrium probability density in phase space. A straightforward implementation of this formula to numerical calculations would be to generate sufficiently many initial conditions $\boldsymbol{\Gamma}(0)$, distributed according to $f(\boldsymbol{\Gamma})$, from which to propagate trajectories of duration $t$. Because of the large number of initial conditions needed, this is in general a very involved calculation, so in practice a different

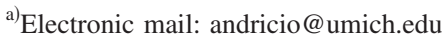

strategy is used. Relying on the ergodic hypothesis, the ensemble average in Eq. (1) is replaced by a time average over a sufficiently long trajectory of a single system,

$$
C(t)=\lim _{T_{\text {tot }} \rightarrow \infty} \frac{1}{T_{\text {tot }}} \int_{0}^{T_{\text {tot }}} A(\boldsymbol{\Gamma}(\tau)) A(\boldsymbol{\Gamma}(t+\tau)) d \tau .
$$

By that means, the same trajectory is used both the generate the initial distribution and to accumulate the correlations. This is usually the method of choice in biomolecular simulations to calculate, for example, orientational correlation functions of bond vectors describing backbone and sidechain mobility in proteins, as measured by NMR relaxation. $^{11}$

However, the calculation of the time average in Eq. (2) is not without problems, either. First, dynamical simulation of biomolecular systems can access relatively short time scales (up to only microseconds at best), thereby limiting the calculation of correlation functions to processes with relaxation times appreciably shorter than this scale. Secondly, and most importantly to the present study, biomolecular systems are characterized by energy landscapes having a broad distribution of barrier heights that often trap a single-molecule trajectory in energy basins. The assumption of ergodicity, which was a requirement to go from Eq. (1) and (2), is thus broken. ${ }^{12}$ Additionally (and obviously), unlike in moleculardynamics simulations of liquids, in simulations of a single biomolecule one lacks the ability to average the timedependent properties over many molecules.

To handle broken ergodicity when calculating thermodynamic averages, (i.e., time-independent averages over a thermodynamic ensemble of a conformation-dependent observable $A(x)$,

$$
\langle A\rangle \propto \int A(x) \exp (-\beta V(x)) d x,
$$

a subset of enhanced-sampling methods have been devised based on a strategy of smoothing out the asperities of the 


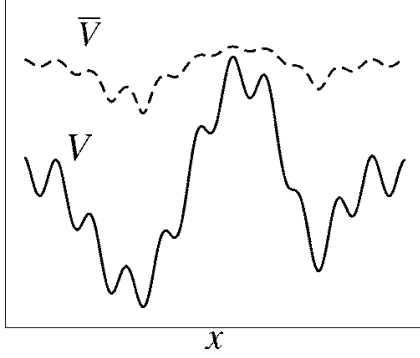

FIG. 1. Caricature depicting the smoothing paradigm. By transforming the potential energy $V$ to a smoother form $\bar{V}$ (that ideally preserves location of stationary points) barriers are effectively lowered, sampling is faster, and the correct thermodynamics is recovered by importance-sampling manipulations. The present work describes a formalism by which kinetics can also be recovered. While more general transformation can be devised, in this first paper we focus on the simple scaling $\bar{V}=\alpha V$.

rugged energy landscape. ${ }^{13}$ By modifying the potentialenergy surface $V \rightarrow \bar{V}$ such that the barriers are effectively lower (see Fig. 1), these methods generate [using molecular dynamics (MD) or Monte Carlo (MC)] samples distributed according to $\exp (-\beta \bar{V})$ without having (in principle) a broken ergodicity problem. Because the samples so generated do not represent the original thermodynamic ensemble, one then retorts to importance-sampling manipulations ${ }^{14}$ to obtain the average corresponding to the untransformed potential. This is done by dividing each value of the accumulated observable $A(x)$ by the "incorrect" weight, $\exp (-\beta \bar{V}(x))$, and by multiplying by the "correct" one, $\exp (-\beta V(x))$. While this strategy recovers the exact thermodynamics, not the same can be said about kinetics. This type of importance-sampling reweighting would not work for the calculation of the timedependent averages of the type $\langle A(0) A(t)\rangle$ in Eq. (1). This is because the entire sequence of events ensuing along a phasespace trajectory that goes from $\boldsymbol{\Gamma}(0)$ to $\boldsymbol{\Gamma}(t)$ would be, on the modified potential surface, different from that on the original potential. The focus of the present paper is to develop a formalism that would allow both using a modified potential and proper accounting for the weight of the entire trajectory such that, in the end, the correct correlation function is recovered even if the computation was done on the transformed potential.

The paper is organized as follows. We begin, in Sec. II, with the theory used to design the formalism. In Sec. III we then test and exemplify the method on a one-dimensional model system, and apply it to the calculation of orientation relaxation of bonds in a dipeptide, with relevance to the experimentally derived, NMR motional parameters. Sec. IV contains a survey of related methods, and we end in Sec. V with a concluding discussion.

\section{THEORETICAL DESIGN OF THE METHOD}

To describe the dynamical trajectory in conformational space, we shall use the Langevin equation,

$m \ddot{x}=-m \gamma \dot{x}+F(x)+\xi(t)$,

with $m$ the mass, $x$ the position, $F(x)=-\nabla V$ the force derived from a potential $V$, and $\xi$ white noise with zero mean and obeying a fluctuation-dissipation relation, $\left\langle\xi(t) \xi\left(t^{\prime}\right)\right\rangle$ $=2 k_{B} \operatorname{Tm} \gamma \delta\left(t-t^{\prime}\right)$, where $T$ is the absolute temperature and $\gamma$ the friction. ${ }^{15}$

The central dynamical object we seek is the conditional probability $P\left(x_{t}, t \mid x_{0}, 0\right)$ to be at $x_{t}$ at time $t$, given that the trajectory started at $x_{0}$ at time $t=0$. It can be obtained from knowledge of the probability $W[\xi(t)]$ of a particular time sequence of random "kicks" $\xi(t)$ and subsequent functional integration over all possible realizations of $\xi(t)$ that conspire to lead to $x_{t}$ at time $t$,

$$
P\left(x_{t}, t \mid x_{0}, 0\right)=\int \mathcal{D} \xi W[\xi] \delta\left(x(t)-x_{t}\right) .
$$

(From here on, the notation convention is that we use square brackets around the function argument of a functional.) Because of the Gaussian nature of the random term, we have that $W[\xi] \propto \exp \left(-\int d t \xi^{2}(t) / 2 w\right)$, with $w=2 m \gamma k_{B} T$. As such, the conditional probability can also be written, using the Wiener formalism of path integrals ${ }^{16}$ as

$$
P\left(x_{t}, t \mid x_{0}, 0\right)=\int_{\left(x_{0}, 0\right)}^{\left(x_{t}, t\right)} \mathcal{D} x|J[x]| \exp \left(-\frac{S[x(t)]}{2 w}\right) .
$$

The functional integration in Eq. (6) is performed over all possible paths connecting the initial and final points. The weight of each trajectory,

$$
W[x(t)] \equiv \exp \left(-\frac{S[x(t)]}{2 w}\right),
$$

is dictated by the action functional,

$$
S[x(t)]=\int_{0}^{t}(m \ddot{x}+m \gamma \dot{x}+\nabla V)^{2} d t^{\prime},
$$

which is referred to as the Onsager-Machlup action ${ }^{17,18}$ (see also Ref. 19). The functional Jacobian $|J[x]|$ (a determinant in the $n$-dimensional case) arises from the $\xi$ to $x$ coordinate transformation and may take different forms depending on the convention adopted to define the path integral. ${ }^{16} \mathrm{~A}$ discretization that is particularly convenient for the scope of the present method is given by the Ito calculus ${ }^{20}$ (a good discussion is also in Ref. 21), for which one can show ${ }^{16}$ that the Jacobian is unity.

Let us now apply, as an enhanced-sampling strategy, a transformation of the potential of type

$$
V \rightarrow \alpha V .
$$

With $0<\alpha<1$, this will scale down the barriers such that, at the same temperature $T$, the rate of exploration of conformational space of a system moving on the $\alpha V$ potential is increased relative to the untransformed potential $V$. For the case of the propagation on the scaled potential $\alpha V$, the weight of an entire trajectory $x(t)$ is simply

$$
W_{\alpha}[x(t)]=\exp \left(-\frac{S_{\alpha}[x(t)]}{2 w}\right),
$$

where $S_{\alpha}=\int(m \ddot{x}+m \gamma \dot{x}+\alpha \nabla V)^{2} d t \quad$ [cf. Eqs. (7) and (8)]. Thus, the correction functional $\Phi_{\text {traj }}$ for the relative weight of a trajectory $x(0 \rightarrow t)$ generated on $V$ that would have passed 
through exactly the same configurations as those corresponding to a trajectory $x_{\alpha}(0 \rightarrow t)$ generated on $\alpha V$ (i.e., $x(t)$ $\equiv x_{\alpha}(t)$ for all times from 0 to $\left.t\right)$ is

$$
\begin{aligned}
& \Phi_{\text {traj }}[x(0 \rightarrow t)]=\frac{W[x(0 \rightarrow t)]}{W_{\alpha}\left[x_{\alpha}(0 \rightarrow t)\right]}=\exp \left(\frac{S_{\alpha}-S}{2 w}\right) \\
& =\exp \left(\frac{\alpha-1}{2 k_{B} T} \int_{0}^{t} \nabla V d x+\frac{\alpha-1}{2 k_{B} \gamma T} \int_{0}^{t} \nabla V d \dot{x}\right. \\
& \left.+\frac{\alpha^{2}-1}{4 k_{B} \gamma T} \int_{0}^{t}(\nabla V)^{2} d t^{\prime}\right)
\end{aligned}
$$

Because the integrals involved in Eq. (12) are not Riemannian, but stochastic (i.e., performed over a trajectory that is continuous everywhere but differentiable nowhere), their value can depend on the discretization definition. For example, care must be taken as to not write $\int_{x(0)}^{x(t)} \nabla V d x=V(t)$ $-V(0)$, unless a midpoint discretization is used in the definition of the integral. Otherwise, as observed by Ito ${ }^{20}$ if a discretization involving the calculation of $\nabla V$ at other points in the finite step interval is applied, additional terms proportional to the second derivative of $V$ appear (see also Ref. 22). An interesting additional subtlety, appreciated by Zuckerman and Woolf, ${ }^{23}$ is that, upon performing the continuum limit of the time slicing (discretization) of the Langevin trajectory, the integration measure $\mathcal{D} x$ was shown,${ }^{24}$ in the case of Stratonovich discretization, to contain a stochastic correction term (Jacobian) that depends on the second derivative of the potential. The omission from the reweighting factor of this term implies, in principle, a deviation from convergence to the equilibrium Boltzmann distribution. However, we have found that this deviation is not detectable in our simulation because of the "background" numerical noise inherent to exponential averaging.

In the numerical implementation, we accumulate the integrands (along each trajectory); as long as $S$ and $S_{\alpha}$ are calculated with the same discretization rule, a reweighting formula [see Eq. (16) and discussion below] should recover the correct average in Eq. (1), corresponding to $V$, over the simulated realizations of the stochastic trajectories on $\alpha V$. We use

$$
\begin{aligned}
& x_{j+1}=x_{j}+c_{1} v_{j} \Delta t-c_{2} \alpha(\nabla V)_{j}(\Delta t)^{2}+\Delta x^{R}, \\
& v_{j+1}=c_{0} v_{j}-c_{1} \alpha(\nabla V)_{j} \Delta t+\Delta v^{R},
\end{aligned}
$$

where the index $j$ counts the time step, and $\Delta x^{R}, \Delta v^{R}$ are random variables with an analytical bivariate distribution ${ }^{19}$ (see also Ref. 10 for details of the algorithm). The numerical coefficients in the above equations are

$c_{0}=\exp (-\xi \Delta t), \quad c_{1}=(\xi \Delta t)^{-1}\left(1-c_{0}\right), \quad c_{2}=(\xi \Delta t)^{-1}\left(1-c_{1}\right)$.

The above algorithm assumes that the systematic force $-\alpha \nabla V(x(t))$ remains approximately constant over each time interval $\Delta t$. This ensures an Ito-type discretization and therefore dispenses with the need to compute the Jacobian.

To calculate the time correlation function of any observable $A(t)$ during a hypothetical propagation on the $V$ poten- tial from an actual evolution on the $\alpha V$ potential, all that is needed is the difference between $S_{\alpha}$ and $S$ so that one can apply a reweighting formula of the type

$$
C(t)=\frac{\sum_{i} A_{i}(0) A_{i}(t) \Phi_{\text {traj }}[x(0 \rightarrow t)]}{\sum_{i} \Phi_{\text {traj }}[x(0 \rightarrow t)]},
$$

where the summation index $i$ labels trajectories originating from canonically distributed phase points corresponding to the untransformed potential. In effect, Eq. (16) is, for kinet$i c s$, the functional equivalent of the usual reweighting scheme employed in umbrella-sampling methods ${ }^{14}$ for thermodynamic averaging. Here, instead of reweighting the conformational point, one reweights the entire functional time dependence of the conformational flow, i.e., the entire trajectory. When the time-average formula for the correlation function, Eq. (2), is to be used, besides this action-functional reweighting, we need an additional factor $\phi_{\text {eq }}$ to correct for the equilibrium distribution $f(\boldsymbol{\Gamma})$ of phase-space points,

$$
\phi_{\mathrm{eq}}(x)=\frac{f(\boldsymbol{\Gamma})}{f_{\alpha}(\boldsymbol{\Gamma})}=\exp \left(\frac{(\alpha-1) V_{0}}{k_{B} T}\right) .
$$

This yields the central formula of our method,

$$
\begin{aligned}
C(t)= & \frac{1}{T_{\text {tot }}-t} \\
& \times \frac{\int_{0}^{T_{\text {tot }}-t} A(\tau) A(t+\tau) \Phi_{\text {traj }}[x(\tau \rightarrow \tau+t)] \phi_{\mathrm{eq}}(x(\tau)) d \tau}{\int_{0}^{T_{\text {tot }}-t} \Phi_{\text {traj }}[x(\tau \rightarrow \tau+t)] \phi_{\mathrm{eq}}(x(\tau)) d \tau},
\end{aligned}
$$

where the summation extends over time frames of a very long trajectory (i.e., one assumed to be ergodic) of duration $T_{\text {tot }} \gg t$.

\section{MODEL SYSTEM EXAMPLES}

In this section, we study two models, of increasing complexity, to test the validity of the method proposed herein, analyze the origins of the errors (numerical noise) inherent to the approach, and provide two strategies for noise reduction.

\section{A. Bistable oscillator system}

A property that is of central interest in many instances involving biomolecular conformational transitions is energy barrier crossing. In calculating correlation functions for complex biomolecules, one feature that poses problems is the fact that, in the simulation, phase points get trapped due to the existence of barriers exceeding thermal energy. A real test of the method should therefore start with model systems with barriers. Consider the Langevin dynamics of a unit-mass particle in a quadratic bistable potential,

$$
V(x)=a x^{2}+b x^{4},
$$

with a frictional coefficient $\gamma=10$ (in arbitrary units). With $a=-3.4$ and $b=1.4$, the two wells of this model potential are 


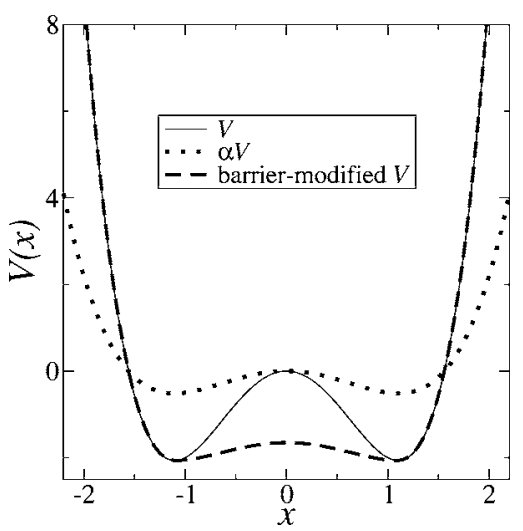

FIG. 2. Bistable potential $V(x)$ : unscaled (continuous line), scaled by $\alpha=1 / 4$ (dotted line), and modified (dashed line) according to formula in Eq (20) [i.e., with only the barrier region scaled by $1 / 4$ (see text)].

separated by a barrier height of approximately two energy units (see Fig. 2).

For a temperature corresponding to a kinetic energy of 2, comparable to the potential barrier, Fig. 3 shows the position-position time autocorrelation functions $C(t)$ $=\langle x(0) x(t)\rangle$ on the unscaled potential in Eq. (19), as well as on potentials scaled by factors of $\alpha=1 / 2$ and $\alpha=1 / 4$, respectively [followed, in the last two cases, by the application of the reweighting formula in Eq. (18)]. All curves were calculated for trajectories of duration $10^{4}$ time units. As expected for this high-temperature limit, because the kinetic energy is sufficient to induce barrier crossing, all calculated correlations match each other.

For connections to more realistic systems, a more stringent test is, undoubtedly, that for which the barrier in the reaction coordinate (i.e., the activation energy) is of significant height compared to $k_{B} T$. In such instances, it takes longer than the typical crossing time to obtain a converged correlation function because one needs to sample enough transitions over the barrier. The gist of the proposed method is to increase the rate of rare events by propagating the trajectory after scaling down the potential. With a lower barrier on the scaled-potential, conformational transitions occur

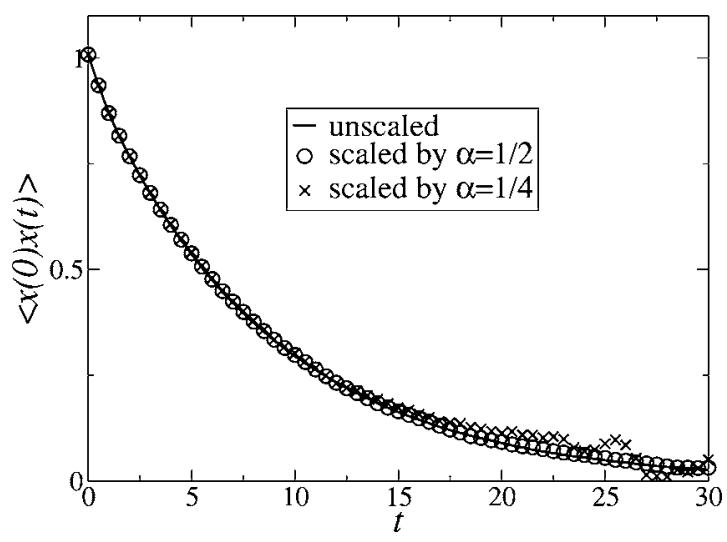

FIG. 3. Position-position time correlation function for bistable potential in Eq. (19), Langevin dynamics simulation with parameters such that the kinetic energy is of the order of the barrier height. Dynamics with scaling factors of $\alpha=1 / 2$ and $1 / 4$, after applying trajectory reweighting, yields same correlation function as that of original system.

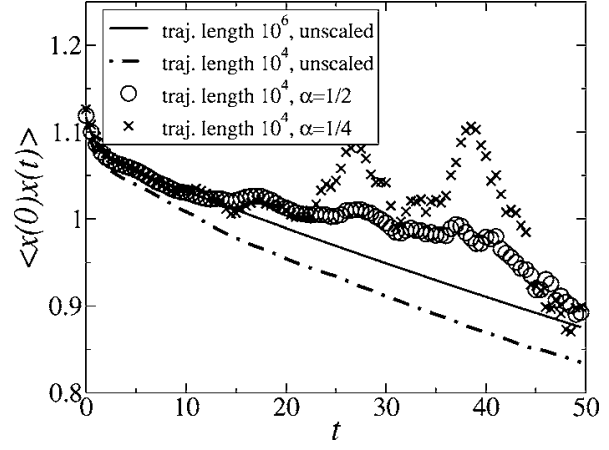

FIG. 4. The low-temperature limit, Langevin trajectory on bistable potential. Shown is the position-position correlation function; parameters such that barrier height is four times larger than thermal energy. As a result, a relatively long trajectory $\left(10^{6}\right.$ time units $)$ is needed to sample both wells and obtain a correlation function that includes interwell excursions. On the other hand, scaling $V \rightarrow \alpha V$ (with two chosen $\alpha$ values labeled in the legend) accelerates interwell hopping so that only $10^{4}$ time units are needed for a nearly-exact calculation. For $\alpha=1 / 4$ the imprecision of the calculation increases for larger times $t$.

more rapidly. Correct correlation functions can then be obtained by reweighting using Eq. (18). The present application to the bistable potential will exemplify the main features of our scaling-reweighting scheme. For the low-temperature limit, we used a kinetic energy of 0.5 , which renders the transitions relatively scarce. We again obtained the correlation functions using scaling factors of $\alpha=1 / 2$ and $1 / 4$. The results are shown in Fig. 4.

We used the time average in Eq. (2) over a trajectory long enough that sufficiently many transitions are experienced. We found that a trajectory of length $10^{6}$ time units was sufficient to reproduce the ensemble average (calculated separately over multiple trajectories of incresing number until convergence, which occured at $10^{6}$ independent starting conditions). Figure 5 shows the time-dependent position on unscaled and scaled potentials. Together with the line-dotted curve in Fig. 4, one can conclude that a total length of $t$ $=10^{4}$ trajectory is not long enough for convergence to a correct correlation function. Along the short trajectory of $10^{4}$ time units, fewer transitions are experienced on the unscaled potential, while many more transitions occur on the scaled potential. Using the short trajectory on the scaled potential and applying the reweighting formula in Eq. (18), one can recover, in principle, the original, exact $C(t)$. Figure 4 summarizes the results. For this system, we were thus able to reduce by two orders of magnitude the time needed for the positional correlation function. The negative aspect of the correlation function as calculated using the reweighting formula and as plotted in Fig. 4 is that, in spite of the fact that the theory is exact, numerical error accumulates for large $t$. This clearly affects the accuracy of the long-time tail calculation, and is rooted in the necessity for overlap of the two types of instantons (see Fig. 5). One deals here with the functional equivalent of the overlap condition required in equilibrium umbrella sampling: if the distribution of states on the original and modified potentials do not overlap significantly, averages pertaining to the original system are not calculated accurately. We return to this important issue first in the next section, where we provide a modified scaling 

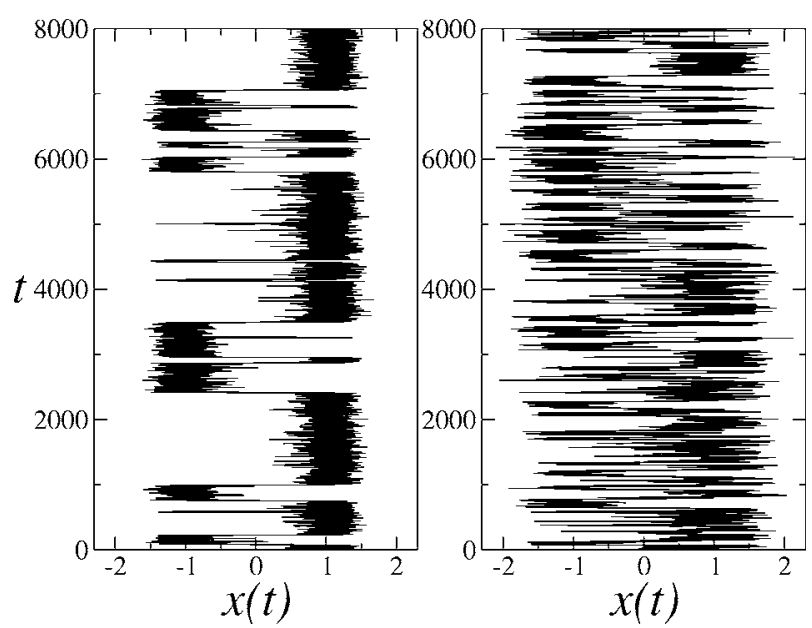

FIG. 5. Two distinct time-dependent position distribution ("instantons") on bistable potential (left, $\alpha=1$ ) and scaled bistable potential (right, $\alpha=1 / 4$ ). Only a portion ( $t$ here in units of time steps) of the low-temperature trajectories used for Fig. 4 is plotted. Although on scaled-potential interwell transitions are more frequent, a broader range of $x$ values is sampled; long- $t$ errors, as evident in Fig. 4, are expected to accrue if enough $x$ overlap between instantons is lacking.

procedure that addresses this aspect, and then in Sec. III C, where we present an analytical derivation of the expected error bounds and propose a time-dependent fitting procedure for noise elimination.

Although of secondary importance when compared to the two orders of magnitude reduction described above, another relative advantage coming from the properties of scaled potential (not used in the present example, though) is that the time step used in the discretization scheme can be larger. The reason for this lies in the fact that the potential is softer, so the fastest time scales (which dictate the maximum size of the time step) will become slower, extending their period by a factor of $1 / \sqrt{\alpha}$.

There are, conceivably, several strategies to distort potentials in a meaningful way such that kinetics is faster. In this first study we have explored the simplest modification: linear scaling. The shifting and scaling procedure (i.e., scaling only in the barrier region) was a modification needed to improve on overall scaling. In future work it will be of interest to explore the relative efficiency of other types of potential scaling, such as logarithmic scaling, ${ }^{13}$ employed by us previously for enhanced equilibrium calculations. An additional point to be made is that, while scaling the potential by $\alpha$ has, roughly, the same effect as raising the temperature, it is important to note that potential scaling provides more flexibility by allowing the scaling of select additive potentialenergy terms (say, the nonbonded or the dihedral terms only). Moreover, scaling functions of increasing sophistication can be conceived, e.g., spatially dependent scaling only along select directions promoting large-scale motion in configuration space ${ }^{25,26}$ ).

For general applications, an optimal compromise between the degree (or the procedure) of smoothing (i.e., in our case, $\alpha$ ) and the growth of the third, error-causing term in the correction factor Eq. (12) will have to be found by preliminary testing.

\section{B. Fine-tuning: A modified, barrier-scaling method}

Figure 4 makes apparent that the reweighting formula, while working well for short-time correlations (up to about $t=25$ ), accumulates significant noise afterwards. Inspecting Eq. (12) and the simulation, we find that one of the reasons for noise accumulation is that the trajectory spends (unnecessary) time at positions far from the barrier region. At those positions, the potential is steep (its derivative is increasingly large) and therefore the positive-definite third term in the argument of the exponential in Eq. (12) (involving the square of the force) keeps growing. This weakens the performance of sample averaging, because exponentially large values occur in the reweighting procedure both in the numerator and denominator. Figure 5 also compares the position distribution in the transformed and untransformed potentials. We can see that the positions on the transformed potential spread more widely in conformation space. The most dynamically interesting part of the energy landscape is the barrier region on a given potential. Positions distributed up in the confining portions of the potential are of less significance to kinetics. To alleviate the noise problem, we propose a modified scaling strategy, which only changes the barrier region of the potential. This approach is akin to the transformation involved in Voter's hyperdynamics ${ }^{27}$ (and in related methods, a discusion of which is postponed until Sec. IV).

By comparing the original and modified scaling strategies (cf. Fig. 2), it is apparent that the modified scaling potential has the same effect in the barrier region, but avoids the negative impact of spreading out in the confining regions. The reweighting formula is still Eq. (18) for the barrier region, and no reweighting is needed when the trajectory is propagated on the original potential. The only modification involves the correction for the initial distribution for the barrier region, which becomes

$$
f(\boldsymbol{\Gamma})=f_{\alpha}(\boldsymbol{\Gamma}) \exp \left(\frac{(\alpha-1)\left(V_{0}+K\right)}{k_{B} T}\right),
$$

where $K$ is a vertical displacement of the transformed potential, needed for a continuous switch between the scaled and original potentials. Absent the wandering about positions far from the barrier region, one is able to calculate the correlation function more efficiently. The modified approach shows, for the low-temperature case, a significant improvement in the long-time region $t>25$, as shown in Fig. 6 .

\section{Bond-vector orientational correlation of alanine dipeptide}

To test the practical utility of the scaling method for biomolecular kinetics, we applied it to alanine dipeptide, $N$-acetylalanyl- $N$-methylamide (see Fig. 7), a model system used extensively in theoretical studies of dynamics and conformational equilibria in proteins. On the one hand, the peptide is small enough to be amenable to kinetical, ${ }^{28}$ thermodynamical, ${ }^{29}$ as well as quantum-mechanical calculations, ${ }^{30}$ and, on the other hand, its backbone configurations are prototypical for the polypeptide backbone of proteins. 


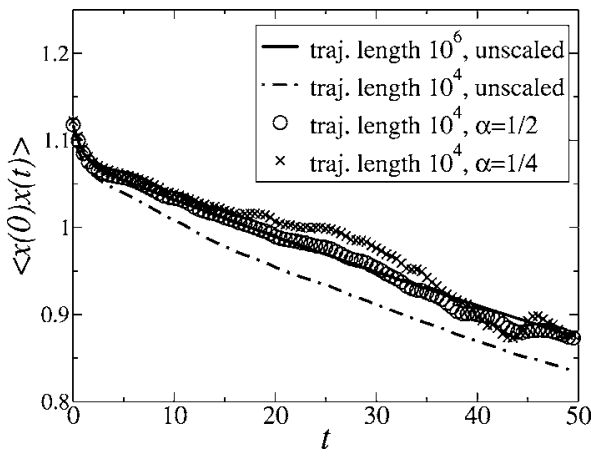

FIG. 6. Correlation function for bistable potential, low-temperature limit, improves accuracy if scaling modified method [according to formula in Eq (20), see also Fig. 2] is used.

As a correlation function to study, we focus on bondorientation time correlation functions. They have recently become of renewed interest in gauging the dynamical aspect of the thermodynamics and kinetics of proteins ${ }^{31}$ due to developments in solution NMR spectroscopy. ${ }^{32,33}$ Backbone $^{34}$ and side-chain dynamics ${ }^{35}$ can be probed using ${ }^{15} \mathrm{~N},{ }^{13} \mathrm{C}$, or ${ }^{2} \mathrm{H}$ relaxation experiments that measure the motion of specific bond vectors in the molecule. The dynamical information from these experiments are often extracted using the LipariSzabo model-free formalism, ${ }^{36}$ which yields so-called $S^{2}$ order parameters, one per bond vector $\mathbf{u}$, quantifying that bond's amplitude of rotational motion, together with the corresponding relaxation times, $\tau$. The order parameters range from 0 (maximum, isotropic motion) to 1 (frozen motion), and are the long-time limit, $S^{2}=\lim _{t \rightarrow \infty} C(t)$, of the autocorrelation function,

$C(t)=\left\langle P_{2}(\mathbf{u}(0) \mathbf{u}(t))\right\rangle=\iint P_{2}\left(\mathbf{u} \cdot \mathbf{u}^{\prime}\right) w^{(2)}\left(\mathbf{u}, \mathbf{u}^{\prime} ; t\right) d \mathbf{u} d \mathbf{u}^{\prime}$,

where $P_{2}(x)$ is the second-order Legendre polynomial and $w^{(2)}$ is the joint probability of observing the unit bond vector at two positions $\mathbf{u}$ and $\mathbf{u}^{\prime}$ at a time separation $t$. The abundance of reports of such motional descriptors for biologically important complexes reveals a rich dynamical behavior on both the subnanosecond as well as the micro-to-millisecod time scales. It has even led to speculations that the functional role of protein dynamics may be encoded in the amino-acid sequence. ${ }^{37}$ Molecular-dynamics simulation is an important tool to complement the measurements because there are several limitations in the relaxation experiments and in their interpretation.

All previous studies comparing experimentally observed to computationally obtained $S^{2}$ parameters $^{38-44}$ employed molecular-dynamics simulation, that, by devise, accurately follows the actual flow in the phase space of the respective biomolecule. However, because for certain motional probes the resulting trajectory was not ergodic, the orientational correlation functions were in some instances shown to have not converged $^{45}$ (particularly for the more mobile bonds). The following simplified example points to the cause of such behavior. Imagine that a particular bond vector samples two states with largely distinct degrees of motion (i.e., with dif-

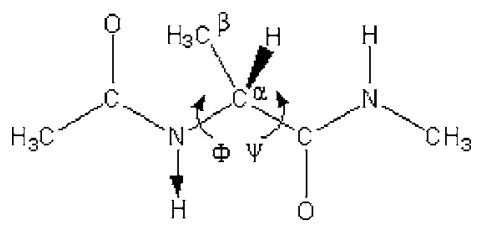

FIG. 7. Chemical structure of alanine dipeptide, with the flexible backbone dihedral angle indicated by the circular arrows. Rotation around $\Phi$ encounters the largest barrier and traps trajectories in two regions, of positive and negative $\Phi$ values, on the nanosecond time scale. $\mathrm{N}-\mathrm{H}$ bond vector studied labeled with straight arrow; its orientational time correlation function, which depends on the correct exploration of both $(+)$ and $(-) \Phi$ regions.

ferent $S^{2}$ and $\tau$ ), and that the two states are conformational macrostates separated by an energy barrier larger than $k T$. A simulation started (and remaining trapped) in one of the macrostates will converge to a $\left(S^{2}, \tau\right)$ pair of values quite different from that observed in a corresponding ensemble experiment.

As a prototype for such an example, we have calculated, for alanine dipeptide, the vectorial form of the reweight formula in Eq. (18), as it pertained to a Langevin trajectory generated using the CHARMM simulation package. ${ }^{46}$ The friction coefficient was $50 \mathrm{ps}^{-1}$, a time step of $1 \mathrm{fs}$ was employed, and the temperature was set to $300 \mathrm{~K}$. To eliminate the six degrees of freedom corresponding to overall tumbling and translation, two atoms (the $C$-terminal atoms $\mathrm{N}$ and $\mathrm{CH}_{3}$ ) were fixed in place. We have used the CHARMM force field with the united-atom, polar-hydrogen parameter set 19 in vacuum. Alanine dipeptide simulations have shown ${ }^{47}$ that rotations around the $\Phi$ backbone dihedral angle are hindered by a high-energy barrier that traps the trajectory on the nanosecond time scale in the positive $\left(\Phi_{+}\right)$and negative $\left(\Phi_{-}\right)$ regions with respect to the values of $\Phi$. We analyze the motional details of the first $\mathrm{NH}$ bond vector from the $N$ terminus; this bond is part of the dihedral $\Phi$ (see Fig. 7). For the purpose of illustrating the instances when the method is expected to yield benefits (i.e., the instances when regular molecular dynamics is expected to fail), we wished to accentuate the stiffness of the bond motion in $\Phi_{+}$. To this end, we added an additional smooth (dihedral-dependent) harmonic term of force constant $500 \mathrm{kcal} / \mathrm{mol} / \mathrm{rad}^{2}$ to each angle that had this particular $\mathrm{NH}$ as one side. This procedure yielded two motional states for the chosen $\mathrm{NH}$ bond vector. When the trajectory is trapped in $\Phi_{+}$, the $\mathrm{NH}$ bond is rigid and converges to $S^{2}=0.82$. When the trajectory is trapped in $\Phi_{-}$, the vector is mobile and yields an $S^{2}$ value below 0.5 . While the angle stiffening modification alters the dynamic and energetic properties of a real alanine dipeptide molecule, it serves, as a model, the purpose of differentiating the order parameters, for our chosen $\mathrm{NH}$ bond, in a manner that is similar to the different order parameters of real proteins: $S^{2}$ values that converge rapidly are often in the $0.8-0.9$ range, while those that do not are typically lower. ${ }^{45}$

Figures 8 and 9 illustrate the calculation of the $\mathrm{NH}$ bond $P_{2}$ correlation function defined in Eq. (21), for various scaling parameters and two trajectory durations. The correlation curves were obtained using the time-average variant of Eq. (21). Two salient features, one favorable and the other less so, are apparent in the plots. Encouragingly, a valid correla- 


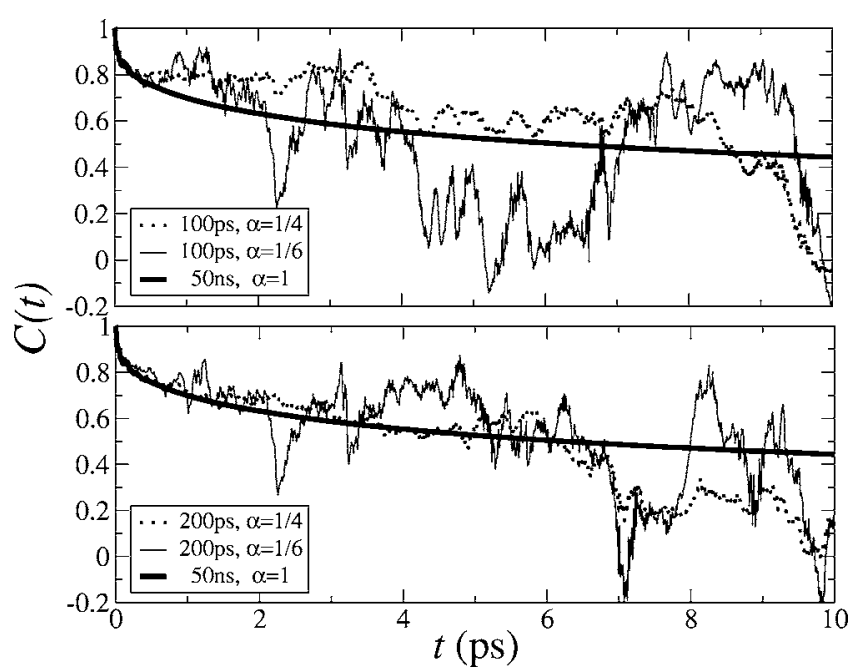

FIG. 8. Dependence on the scaling factor $\alpha$ for the orientational correlation function $C(t)=\left\langle P_{2}(\mathbf{u}(0) \mathbf{u}(t))\right\rangle$ of an $\mathrm{N}-\mathrm{H}$ bond in alanine dipeptide, $T$ $=300 \mathrm{~K}$ for original and scaled-potential trajectories of the same duration: $100 \mathrm{ps}$ (upper panel) and $200 \mathrm{ps}$ (lower panel). The "exact" result on the unscaled potential is shown (thick line, $\alpha=1$ ) based on 50 ns trajectory.

tion function (i.e., one that matches that from an ergodic trajectory) is obtained by carrying out the scalingreweighting scheme on a time scale on which regular Langevin MD is non ergodic and converges to an incorrect function. However, as was for the bistable oscillator, error accumulation is again significant for large values of $t$. Similarly to that one-dimensional case, it increases with $t$. We address the cause behind this issue and propose approaches to filter out the error based on an estimate of the timedependent standard deviation of $C(t)$ in the next section. For the particular case at hand, bond-vector relaxation for internal dynamics occuring on two-time scales can be described by an extended Lipari-Szabo formalism, ${ }^{48}$

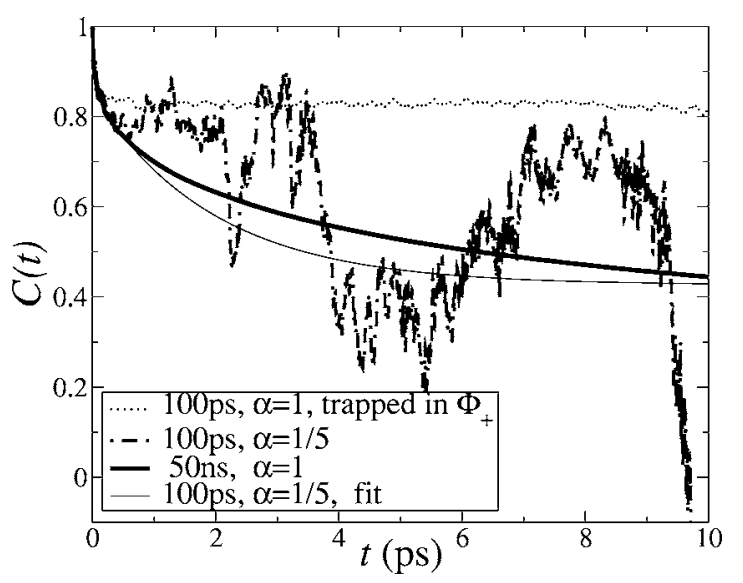

FIG. 9. Fit of extended Lipari-Szabo formalism to orientational correlation function $C(t)=\left\langle P_{2}(\mathbf{u}(0) \mathbf{u}(t))\right\rangle$ of $\mathrm{N}-\mathrm{H}$ bond in alanine dipeptide, $T=300 \mathrm{~K}$ for a scaled-potential trajectory of $100 \mathrm{ps}$ with $\alpha$. The noise-reduced curve (see legend) is obtained by fitting to Eq. (22) of the generalized order parameter formalism (see text); obtained motional parameters are $S^{2}=0.42$, $S_{f}^{2}=0.85, \tau_{f}=50 \mathrm{fs}$, and $\tau_{s}=2.0 \mathrm{ps}$. Using the fit of the reweighted correlation propagated on scaled potential, one can obtain, more than two orders of magnitude faster, nearly the same correlation function as that obtained in the $50 \mathrm{~ns}$ needed for convergence by regular Langevin molecular dynamics. If regular dynamics is started in the $\Phi_{+}$region and propagates for only $100 \mathrm{ps}$, it remains trapped and an erroneous correlation (dotted line) is obtained.

$$
C(t)=S^{2}+\left(1-S_{f}^{2}\right) e^{-t / \tau_{f}}+\left(S_{f}^{2}-S^{2}\right) e^{-t / \tau_{s}},
$$

where $\tau_{f}$ and $\tau_{s}$ are two independent time constants for the fast and slow internal motions, respectively, and $S_{f}^{2}$ and $S_{s}^{2}$ are corresponding order parameters, with the generalized order parameter being $S^{2}=S_{f}^{2} S_{s}^{2}$. The result of fitting to Eq. (22) the noisy correlation obtained by the scaling method is presented in Fig. 9. As seen, the result of applying the scaleand-reweight strategy on a trajectory of merely 100 ps is a good approximation for the "exact" correlation function calculated by extensive sampling over 50 ns.

\section{Error analysis for overdamped Langevin motion}

It is illuminating to study the high-friction limit of the Langevin equation. Because protein motion on time scales beyond $1 \mathrm{ps}$ is overdamped, ${ }^{49}$ this limit yields a good approximation for biomolecular conformational transitions. The simplification will bring insight into the limitation of the reweighting scheme employed herein, and will explain the error observed in the long-time tail of the NH bond autocorrelation function in Fig. 8. For the case of an overdamped Langevin equation, written for convenience in one dimension,

$$
m \gamma \dot{x}=F(x)+\xi(t),
$$

in numerical implementations, on $V$ and $\alpha V$, we use a propagation scheme,

$$
x_{j+1}=x_{j}+\frac{F_{j} \Delta t}{m \gamma}+\Delta x_{j}^{R},
$$

and, respectively,

$$
x_{\alpha, j+1}=x_{\alpha, j}+\frac{\alpha F_{j} \Delta t}{m \gamma}+\Delta x_{\alpha, j}^{R},
$$

where, at step $j, \Delta x^{R}$ and $\Delta x_{\alpha}^{R}$ are Gaussian random displacements, i.e., distributed according to

$$
w_{j}\left(\Delta x^{R}\right)=\frac{1}{\sqrt{2 \pi} \sigma} \exp \left(-\frac{\left(\Delta x^{R}\right)^{2}}{2 \sigma^{2}}\right) \text {. }
$$

Denoting by $s_{j}=F_{j} \Delta t /(m \gamma)$ the systematic part of the discrete, stepwise propagation, and requiring that $x_{\alpha, j}=x_{j}$ for all $j$, we have that the correction factor for each step is

$$
\frac{w_{j}\left(\Delta x^{R}\right)}{w_{j}\left(\Delta x_{\alpha}^{R}\right)}=\exp \left(\frac{2(1-\alpha) s \Delta x-\left(1-\alpha^{2}\right) s^{2}}{2 \sigma^{2}}\right),
$$

where $\Delta x=x_{j+1}-x_{j}$. The multiplication of the factors for all steps $j$ of a trajectory yields the trajectory's total correction factor,

$$
\begin{aligned}
\frac{W[x]}{W_{\alpha}\left[x_{\alpha}\right]} & =\prod_{j} \frac{w_{j}\left(\Delta x^{R}\right)}{w_{j}\left(\Delta x_{\alpha}^{R}\right)} \\
& =\exp \left[\frac{1}{2 \sigma^{2}} \sum_{j}\left(2(1-\alpha) s \Delta x-\left(1-\alpha^{2}\right) s^{2}\right)\right] .
\end{aligned}
$$

In the continuum limit, it can be shown that this converges to 


$$
\frac{W[x]}{W_{\alpha}\left[x_{\alpha}\right]}=\exp \left(\frac{\alpha-1}{2 k_{B} T} \int \nabla V d x+\frac{\alpha^{2}-1}{4 k_{B} \gamma T} \int(\nabla V)^{2} d t\right),
$$

a particular case of Eq. (12). However, even before taking the limit, analysis of Eq. (28) reveals a couple of interesting features. Firstly, the term $\left(1-\alpha^{2}\right) s^{2} \equiv \zeta$ in the argument of the exponential, which is the largest source of the long-time error observed in Figs. 4 and 8, can be thought of as a random variable, since $s$ itself can be regarded as a random variable: $\left.s_{j}=\left(\Delta x_{\alpha, j}^{R}-\Delta x_{j}^{R}\right) /(1-\alpha)\right)$. In the limiting case that $\Delta x_{j}^{R}$ and $\Delta x_{\alpha, j}^{R}$ are independent random variables, the variance of their difference is simply the sum of their variances, so the variance of $s$ is $\sigma_{s}^{2}=2 \sigma^{2} /(1-\alpha)^{2}$. The sum over $\zeta$ involved in Eq. (28) is an increasing function of the total trajectory time $\tau$. It now gives the dominant correction factor, $\exp \left(\sum_{j=1}^{t} \zeta\right)$. For large $t$, it can cause significant overflow errors when the reweighting formula, Eq. (16) or (18), are used. One can show that the average of $\zeta$ is exactly $\langle\zeta\rangle=1+\alpha /(1-\alpha)$. One could therefore define a new variable, $\eta=\zeta-\langle\zeta\rangle$, and analytically factor out $\exp (t\langle\zeta\rangle)$ both in the numerator and denominator of Eq. (16) or (18). By simplification, this will yield a correction factor that involves the exponential of a (random) quantity, $\mathcal{E}=\Sigma \eta$, with average zero. However, according to the central limit theorem, the standard deviation of $\mathcal{E}$ will still grow as the $\sqrt{t}$. Therefore, a typical correction factor is expected to lie anywhere between zero and an upper bound that keeps growing as $\exp (\mathcal{C} \sqrt{t})$, with $\mathcal{C}$ a constant. This is the reason for the significant error inherent to the basic scaling approach; the average of an exponential is a notoriously noisy and biased statistic. To filter out this noise for the case of the alanine dipeptide $\mathrm{NH}$ correlation in Sec. III C, we have used fitting to a generalized model for the internal dynamics on two disparate time scales. For general application where such knowledge is lacking, one can use a moving average with an averaging window width increasing with $t$ (to account for the increase of the standard deviation). In future work, we shall explore the noise properties of more sophisticated smoothing schemes in combination with noise elimination based on the Wiener-Khintchine theory and related signal processing techniques.

\section{CONNECTIONS TO EXISTING TRAJECTORY SAMPLING STRATEGIES}

The Onsager-Machlup action $S$ is central to several recent developments that use multiple trajectories (or paths) connecting two conformational states. The weight of paths according to the action, $\exp (-S)$, allows one, in the transition path sampling method of Chandler and co-workers ${ }^{50,51}$ (see also Ref. 52), to exploit the isomorphism between the distribution of paths and the canonical distribution of conformations of a polymer. This therefore is inducive to the design of importance-sampling (Monte Carlo or related) algorithms for kinetic calculations derived in analogy to polymer Monte Carlo methods. Using a related approach, Geissler and Chandler ${ }^{53}$ have used importance sampling in trajectory space to calculate nonequilibrium solvent response functions. Also, the transition path sampling method has been applied with promising success to a variety of biomolecular systems. ${ }^{54,55}$ In an equally exciting related development, the stochastic difference equation method of Elber et al. for long-time dynamics ${ }^{21}$ uses a minimization of a discretized version of $S$ with impressive speedup of the propagation of the equations of motion for proteins, with time steps that are two orders of magnitude longer than regular dynamics usually employs. Both of the above approaches have a diffusivelimit method as particular case. ${ }^{56,57}$

The reweighting procedure outlined in the present paper is related to Wagner's approach ${ }^{58}$ to variance reduction (importance sampling being a subcase of variance reduction). Woolf $^{59}$ and Zuckerman and Woolf ${ }^{60}$ adapted Wagner's approach and designed a dynamical importance-sampling method for the calculation of reaction rates. Given the knowledge of a target state, Langevin dynamics was augumented with a biasing vector pointing to that target. Similar dynamical importance sampling has been used by Mazonka et $a l .{ }^{61}$ and by Zou et al. ${ }^{62}$ While the smoothing and reweighting uses in principle the same strategy as dynamical importance sampling, it differs in two aspects. It does not require knowledge of two end points and can therefore be used as a general method for calculating any time correlation function whose convergence depends on visiting all relevant basins during the course of the simulation. Secondly, it uses a time average over a single trajectory and is therefore amenable to the calculation of biomolecular time correlation functions.

To generate multiple trajectories for the study of the rate of activated processes [i.e., using the ensemble average in Eq. (16)], action reweighting was also used by Chen et al., ${ }^{63}$ who have recently presented an intriguing method that resolves conformational changes by dividing a rare-transition event, taking place from an initial time 0 to a final time $t_{f}$, into two parts: an activation half-path, from time 0 to an intermediate time $t_{M}$, which is governed by a Langevin equation with negative friction $(\gamma<0)$, followed by a deactivation path during $\left(t_{M}, t_{f}\right)$ governed by the regular, positive friction.

In the context of altering the energy surface to obtain kinetic properties faster, the smoothing suggested in the present paper is related to the hyperdynamics method of Voter, ${ }^{27,64}$ who calculates transition-state-theory rate constants, using importance-sampling manipulations, after adding a boost potential that is zero at the transiton state. The work of Hamelberg et al. ${ }^{65}$ is along the same line. While it dispenses with the need to calculate eigenvalues of the Hessian, it requires an extrapolation to zero boost, from several simulations, to obtain kinetic data. Because both approaches involve, in effect, an energy-smoothing transformation $\bar{V}$ $=\bar{V}(x)$ (i.e., a function of coordinates only), a generalization of the reweighting factor, Eq. (12), for the case of the systematic force $-\nabla \bar{V}$ should be straightforward. It would enable one to calculate directly entire correlation functions, and thereby to relate them to the kinetics that hyperdynamics is computing. Reference 66 contains a good review of these and other accelerated dynamics methods. While the idea of modifying potentials to compute more efectively time corre- 
lation functions is newly introduced in the present work, modifying the Hamiltonian to sample different trajectories is fundamental to all the accelerated dynamical strategies described above, as well to several other related approaches (that construct reactive pathways using ad hoc probability functionals ${ }^{67-72}$ ). These methods are covered in the reviews of Dellago et al., ${ }^{73}$ Elber et al., and ${ }^{74}$ Elber $^{75}$.

In future work, it would also be of interest to explore the application of potential scaling to sampling an ensemble of transition trajectories. For example, in the case of the stochastic difference equation method, scaling would allow taking even larger time steps. In another example, for transition path sampling, scaling the potential could induce enhanced conformational sampling in path space. With the energy landscape replaced by the action landscape, large barriers (or ridges $^{76}$ ) of action can cause broken ergodicity in trajectory space, and it has been realized that a straightforward integration over all significant trajectories can become challenging. It should also be worthwhile to use scaling-reweighting approaches in combination with parallel-replica dynamical methods of the type developed by Voter ${ }^{77}$ and further refined by Shirts and Pande, ${ }^{78}$ who have extended dynamical simulations of protein folding in the microsecond range. As the effect of applying the two methods (scaling and parallel replica dynamics) in concert is expected to be multiplicative, this may make feasible the calculation of submillisecond correlations that are descriptive of the large-amplitude, collective motions of biomolecular systems.

While correlations for local conformational dynamics currently within the range of regular simulations decay on the 100 ps time scales, experiments both for proteins ${ }^{79}$ and DNA $^{80}$ reveal complex dynamics on multiple time scales that extend beyond the nanosecond time scale. Moreover, novel experiments based on residual dipolar couplings extend to even longer the time scale of relaxation probed by NMR. ${ }^{81}$ Novel dynamical algorithms of the type presented here should be useful therefore if one wishes to simulate such slow conformational kinetics.

\section{CONCLUSIONS}

We have presented and analyzed a method of general applicability to the efficient calculation of correlation functions dominated by long-time conformational transitions. The method generates a Langevin trajectory on a potential that is scaled (such that barriers are effectively lowered) and then applies to the trajectory, a functional reweighting scheme based on stochastic path-integral theory. Accordingly, one recovers, in principle, the exact correlation function corresponding to the original system (i.e., with unscaled potential). Because exploration on the scaled potential is faster, one can calculate efficiently, and with general applicability, time correlation functions of any conformational property. We have considered two applications, a bistable oscillator and a model peptide. They have exemplified the strenghts and weaknesses expected of the approach when applied to models of molecular conformational transitions. The advantage of a modified scaling strategy that scales the barrier regions only was also addressed. We have also ana- lyzed the errors associated with the method, have provided an estimate of their time-dependent standard deviation, and have employed a two-time-scale relaxation mechanism to fit time correlation functions for bond-vector orientations. Using the protein model system, we have showed that time correlations for peptide bond motions can be calculated more than two orders of magnitude faster than regular molecular dynamics.

\section{ACKNOWLEDGMENT}

This work was supported by funds from the University of Michigan.

${ }^{1}$ R. Kubo, J. Phys. Soc. Jpn. 12, 570 (1957).

${ }^{2}$ D. Chandler, Introduction to Modern Statistical Mechanics (Oxford University Press, New York, 1987).

${ }^{3}$ B. J. Berne and R. Pecora, Dynamic Light Scattering (Wiley, New York, 1976).

${ }^{4}$ R. L. Fulton, J. Chem. Phys. 55, 1386 (1971).

${ }^{5}$ R. G. Gordon, Adv. Magn. Reson. 3, 1 (1968).

${ }^{6}$ U. M. Titulaer and J. M. Deutch, J. Chem. Phys. 60, 1502 (1974).

${ }^{7}$ T. Moriya, Prog. Theor. Phys. 28, 371 (1962).

${ }^{8}$ T. J. Chuang and K. B. Eisenthal, J. Chem. Phys. 57, 5094 (1972).

${ }^{9}$ H. Mori, Prog. Theor. Phys. 33, 423 (1965).

${ }^{10}$ M. P. Allen and D. J. Tildesley, Computer Simulation of Liquids (Oxford University Press, New York, 1987).

${ }^{11}$ D. C. Chatfield, A. Szabo, and B. R. Brooks, J. Am. Chem. Soc. 120, 5301 (1998)

${ }^{12}$ R. Palmer, Adv. Phys. 31, 669 (1982).

${ }^{13}$ I. Andricioaei and J. E. Straub, J. Chem. Phys. 107, 9117 (1997)

${ }^{14}$ G. M. Torrie and J. P. Valleau, J. Comput. Phys. 23, 187 (1977).

${ }^{15}$ While the notation is one dimensional, it is straightforward to extend the treatment to $n$ dimensions. In general $\gamma_{i j}$ is a tensor and, additionally, for large molecules, might vary with the distance from the molecular surface, (see Ref. 82) However, for the purpose of developing the method, these issues can be neglected without loss of generality. Moreover, the formalism can be extended to Langevin equations with memory (i.e., when the noise is colored).

${ }^{16}$ H. Kleinert, Path Integrals in Quantum Mechanics, Statistics, Polymer Physics and Financial Markets, 3rd ed. (World Scientific, Singapore 2004).

${ }^{17}$ L. Onsager and S. Machlup, Phys. Rev. 91, 1505 (1953).

${ }^{18}$ S. Machlup and L. Onsager, Phys. Rev. 91, 1512 (1953).

${ }^{19}$ S. Chandrasekhar, Rev. Mod. Phys. 15, 1 (1943).

${ }^{20} \mathrm{~K}$. Ito, Mem. Am. Math. Soc. 4, 1, (1951).

${ }^{21}$ R. Elber, J. Meller, and R. Olender, J. Phys. Chem. B 103, 899 (1999).

${ }^{22}$ L. Schulman, Techniques and Applications of Path Integration (Wiley, New York, 1981).

${ }^{23}$ D. M. Zuckerman and T. B. Woolf, Phys. Rev. E 6302, 016702 (2001).

${ }^{24}$ R. Stratonovich, Selected Translations in Mathematical Statistics and Probability (American Mathematical Society, Providence, RI, 1971), Vol. 10 , p. 273

${ }^{25}$ I. Andricioaei, A. R. Dinner, and M. Karplus, J. Chem. Phys. 118, 1074 (2003).

${ }^{26}$ J. MacFadyen and I. Andricioaei, A skewed momenta method to efficiently generate conformational transition trajectories, J. Chem. Phys. 1237 (2005).

${ }^{27}$ A. F. Voter, Phys. Rev. Lett. 78, 3908 (1997).

${ }^{28}$ R. J. Loncharich, B. R. Brooks, and R. W. Pastor, Biopolymers 32, 523 (1992).

${ }^{29}$ D. J. Tobias and C. L. Brooks III, J. Phys. Chem. 96, 3864 (1992).

${ }^{30}$ T. Head-Gordon, M. Head-Gordon, M. J. Frisch, C. L. Brooks III, and J. A. Popple, J. Am. Chem. Soc. 113, 5989 (1991).

${ }^{31}$ J. D. Forman-Kay, Nat. Struct. Biol. 6, 1086 (1999).

${ }^{32}$ A. G. Palmer, Chem. Rev. (Washington, D.C.) 104, 3623 (2004).

${ }^{33}$ H. J. Dyson and P. E. Wright, Chem. Rev. (Washington, D.C.) 104, 3607 (2004).

${ }^{34}$ A. M. Mandel, M. Akke, and A. G. Palmer, Biochemistry 35, 16009 (1996).

${ }^{35}$ D. R. Muhandiram, T. Yamazaki, B. D. Sykes, and L. E. Kay, J. Am. 
Chem. Soc. 117, 11536 (1995)

${ }^{36}$ G. Lipari and A. Szabo, J. Am. Chem. Soc. 104, 4546 (1982).

${ }^{37}$ A. J. Wand, Nat. Struct. Biol. 8, 926 (2001).

${ }^{38}$ Y. Pang, M. Buck, and E. R. P. Zuiderweg, Biochemistry 41, 2655 (2002)

${ }^{39}$ H. Schwalbe, K. M. Fiebig, M. Buck, J. A. Jones, S. B. Grimshaw, A. Spencer, S. J. Glaser, L. J. Smith, and C. M. Dobson, Biochemistry 36 8977 (1997).

${ }^{40}$ M. Philippopoulos and C. Lim, J. Mol. Biol. 254, 771 (1995).

${ }^{41}$ L. J. Smith, A. E. Mark, C. M. Dobson, and W. F. Vangunsteren, Biochemistry 34, 10918 (1995).

${ }^{42}$ P. E. Smith, R. C. Vanschaik, T. Szyperski, K. Wuthrich, and W. F. Vangunsteren, J. Mol. Biol. 246, 356 (1995).

${ }^{43}$ A. G. Palmer and D. A. Case, J. Am. Chem. Soc. 114, 9059 (1992).

${ }^{44}$ R. M. Levy, C. M. Dobson, and M. Karplus, Biophys. J. 39, 107 (1982).

${ }^{45}$ D. A. Case, Acc. Chem. Res. 35, 325 (2002).

${ }^{46}$ B. R. Brooks, R. E. Bruccoleri, B. D. Olafson, D. J. States, S. Swaminathan, and M. Karplus, J. Comput. Phys. 4, 187 (1983).

${ }^{47}$ P. Smith, B. M. Pettitt, and M. Karplus, J. Phys. Chem. 97, 6907 (1993).

${ }^{48}$ G. M. Clore, A. Szabo, A. Bax, L. E. Kay, P. C. Driscoll, and A. M. Gronenborn, J. Am. Chem. Soc. 112, 4989 (1990).

${ }^{49}$ J. C. Smith, Q. Rev. Biophys. 24, 227 (1991).

${ }^{50}$ C. Dellago, P. G. Bolhuis, F. S. Csajka, and D. Chandler, J. Chem. Phys. 108, 1964 (1998).

${ }^{51}$ C. Dellago, P. G. Bolhuis, and D. Chandler, J. Chem. Phys. 110, 6617 (1999).

${ }^{52}$ L. R. Pratt, J. Chem. Phys. 85, 5045 (1986).

${ }^{53}$ P. L. Geissler and D. Chandler, J. Chem. Phys. 113, 9759 (2000).

${ }^{54}$ P. G. Bolhuis, Proc. Natl. Acad. Sci. U.S.A. 100, 12129 (2003).

${ }^{55}$ R. Radhakrishnan and T. Schlick, Proc. Natl. Acad. Sci. U.S.A. 101, 5970 (2004)

${ }^{56}$ A. K. Faradjian and R. Elber, J. Chem. Phys. 120, 10880 (2004).

${ }^{57}$ D. Moroni, P. G. Bolhuis, and T. S. van Erp, J. Chem. Phys. 120, 4055 (2004).

${ }^{58}$ W. Wagner, J. Comput. Phys. 71, 21 (1987).
${ }^{59}$ T. B. Woolf, Chem. Phys. Lett. 289, 433 (1998).

${ }^{60}$ D. M. Zuckerman and T. B. Woolf, J. Chem. Phys. 111, 9475 (1999).

${ }^{61}$ O. Mazonka, C. Jarzynski, and J. Blocki, Nucl. Phys. A 650, 499 (1999).

${ }^{62}$ G. Zou, R. D. Skeel, and S. Subramaniam, Biophys. J. 79, 638 (2000).

${ }^{63}$ L. Y. Chen, S. C. Ying, and T. Ala-Nissila, Phys. Rev. E 65, 042101 (2002).

${ }^{64}$ A. F. Voter, J. Chem. Phys. 106, 4665 (1997).

${ }^{65}$ D. Hamelberg, T. Shen, and J. A. McCammon, J. Chem. Phys. 122, 241103 (2005).

${ }^{66}$ A. F. Voter, F. Montalenti, and T. C. Germann, Annu. Rev. Mater. Res. 32, 321 (2002).

${ }^{67}$ R. Elber and M. Karplus, Chem. Phys. Lett. 139, 375 (1987).

${ }^{68}$ R. E. Gillilan and K. R. Wilson, J. Chem. Phys. 97, 1757 (1992).

${ }^{69}$ E. M. Sevick, A. T. Bell, and D. N. Theodorou, J. Chem. Phys. 98, 3196 (1993).

${ }^{70}$ S. H. Huo and J. E. Straub, J. Chem. Phys. 107, 5000 (1997).

${ }^{71}$ G. Henkelman, B. P. Uberuaga, and H. Jonsson, J. Chem. Phys. 113, 9901 (2000).

${ }^{72}$ D. Passerone, M. Ceccarelli, and M. Parrinello, J. Chem. Phys. 118, 2025 (2003).

${ }^{73}$ C. Dellago, P. G. Bolhuis, and P. L. Geissler, Adv. Chem. Phys. 123, 1 (2002).

${ }^{74}$ R. Elber, A. Ghosh, and A. Cardenas, Acc. Chem. Res. 35, 396 (2002).

${ }^{75}$ R. Elber, Curr. Opin. Struct. Biol. 15, 151 (2005).

${ }^{76}$ P. G. Bolhuis, D. Chandler, C. Dellago, and P. L. Geissler, Annu. Rev. Phys. Chem. 53, 291 (2002).

${ }^{77}$ A. F. Voter, Phys. Rev. B 57, R13985 (1998).

${ }^{78}$ M. R. Shirts and V. S. Pande, Phys. Rev. Lett. 86, 4983 (2001).

${ }^{79}$ X. S. Xie, J. Chem. Phys. 117, 11024 (2002).

${ }^{80}$ E. B. Brauns, M. L. Madaras, R. S. Coleman, C. J. Murphy, and M. A. Berg, Phys. Rev. Lett. 88, 158101 (2002).

${ }^{81}$ W. Peti, J. Meiler, R. Bruschweiler, and C. Griesinger, J. Am. Chem. Soc. 124, 5822 (2002)

${ }^{82}$ W. Doster, S. Cusack, and W. Petry, Nature (London) 337, 754 (1989). 